\title{
Hiding in plain sight: latent grouping factors in linear models
}

\author{
Dr. Chris Franck \\ Department of Statistics \\ Virginia Tech, USA
}

Standard linear modeling approaches make potentially simplistic assumptions regarding the structure of categorical effects that may obfuscate more complex relationships governing data. For example, recent work focused on the two-way unreplicated layout has shown that hidden groupings among the levels of one categorical predictor frequently interact with the ungrouped factor. In this talk, I extend the notion of a "latent grouping factor" to linear models in general. The proposed work allows researchers to determine whether an apparent grouping of the levels of a categorical predictor reveals a plausible hidden structure given the observed data. Specifically, I offer a Bayesian model selection-based approach to reveal latent group-based heteroscedasticity, regression effects, and/or interactions. Failure to account for such structures can produce misleading conclusions. Since the presence of latent group structures is frequently unknown $a$ priori to the researcher, I use fractional Bayes factor methods and mixture $g$-priors to overcome lack of prior information. 